\title{
Optimal Management of Public Perceptions During A Flu Outbreak: A Game-Theoretic Perspective
}

\author{
Aniruddha Deka ${ }^{1} \cdot$ Buddhi Pantha ${ }^{2}$ Samit Bhattacharyya ${ }^{1}$
}

Received: 24 September 2019 / Accepted: 2 October 2020 / Published online: 16 October 2020

(c) Society for Mathematical Biology 2020

\begin{abstract}
Public perceptions and sentiments play a crucial role in the success of vaccine uptake in the community. While vaccines have proven to be the best preventive method to combat the flu, the attitude and knowledge about vaccines are a major hindrance to higher uptake in most of the countries. The yearly coverage, especially in the vulnerable groups in the population, often remains below the herd immunity level despite the Flu Awareness Campaign organized by WHO every year worldwide. This brings immense challenges to the nation's public health protection agency for strategic decision-making in controlling the flu outbreak every year. To understand the impact of public perceptions and vaccination decisions while designing optimal immunization policy, we model the individual decision-making as a two-strategy pairwise contest game, where pay-off is considered as a function of public health effort for the campaign. We use Pontryagin's maximum principle to identify the best possible strategy for public health to implement vaccination and reduce infection at a minimum cost. Our optimal analysis shows that the cost of public health initiatives is qualitatively and quantitatively different under different public perceptions and attitudes towards vaccinations. When individual risk perception evolves with vaccine uptake or disease induced death, our model demonstrates a feed-forward mechanism in the dynamics of vaccination and exhibits an increase in vaccine uptake. Using numerical simulation, we also observe that the optimal cost can be minimized by putting the effort in the beginning and later part of the outbreak rather than during the peak. It confers that public health efforts towards disseminating disease severity or actual vaccination risk might accelerate the vaccination coverage and mitigate the infection faster.
\end{abstract}

Keywords Vaccination · Herd immunity · SIR model · Free-riding behaviour . Evolutionary game theory $\cdot$ Pontryagin's maximum principle 


\section{Introduction}

Public health department of every country faces several challenges-economical, infrastructure, epidemiological, political and social - to prevent and control influenza every year. Among these, individual or patient-level perceptions about vaccinations are paramount. Despite the flu awareness program each year by the World Health Organization (WHO), influenza vaccination coverage among high-risk groups has nonetheless been declining in a number of countries, especially, in the WHO European Region in recent years (Jones 2012). Local beliefs, lack of trust in the health care system, misperception about the probability of infection, indigenous health practices to avoid or treat influenza and such other factors result in low vaccination coverage (Bukhsh et al. 2018; Strelitz et al. 2015). Individuals decide vaccination only when they perceive high levels of risk from disease; otherwise, they are more likely to evade vaccination, even if vaccines are available at the beginning of the outbreak (Arda et al. 2011; Li et al. 2012; Shahrabani and Benzion 2010; Seale et al. 2010; Baguelin et al. 2013; Shim 2013). A recent study by Center for Disease Control and Prevention (CDC), USA, found out persistence of many barriers to vaccine acceptance in public including healthcare workers (HCW) — some of which may include limited understanding of influenza and immunization recommendations, indications of greater subgroup recognition of the value of flu vaccination, continued resistance to vaccinations, and overestimation of the effectiveness of non-vaccine measures, and many other religious misperceptions (Nowak et al. 2015). This, however, incurs a huge economic burden on the nation. Previous experience such as $(\mathrm{H} 1 \mathrm{~N} 1,2009)$ pandemics has demonstrated substantial health and economic loss across the world (Donaldson et al. 2009). According to the WHO estimates, worldwide annual epidemics are estimated to result in about 3 to 5 million cases of severe illness, and about 290,000 to 650,000 respiratory deaths (Danielle Iuliano et al. 2018; GBD 2018).

Classical game theory provides a framework to understand such strategic interactions between epidemiology and human vaccinating behaviour under voluntary vaccination policy. Behaviour-prevalence models based on evolutionary game theory have shown how 'herd immunity' can lead to 'free-riding' behaviour in voluntary vaccination game. Studies have considered the evolution of strategies through imitation dynamics to show how successful strategies are adopted by members of the population (Bauch 2005; d'Onofrio et al. 2012; Bhattacharyya and Bauch 2012, 2011; Galvani et al. 2007; Reluga et al. 2006; Reluga 2010; Reluga and Galvani 2011; d'Onofrio et al. 2007, 2011). On the other hand, there are ample modelling works that highlight on the dynamic vaccination during seasonal influenza and pandemic (Bowman et al. 2011; Baguelin et al. 2012; Mercer et al. 2011; Basta et al. 2009), considering strategic allocation of vaccination such as the time when and how long vaccination should be continued, identifying specific age class in population, optimal distribution of vaccines for minimizing infection and severe outcomes from it such as deaths or hospitalization (Bowman et al. 2011; Matrajt and Longini Jr 2010; Patel et al. 2005; Verriest et al. 2005; Gaff and Schaefer 2009; Medlock and Meyers 2009; Lee et al. 2012; Ullah et al. 2012). Such works, in a more theoretical context, characterize the complexity of dynamic vaccination, frame as an optimal control problem and solve the optimality system indicating its type and possible singularities. However, optimizing 
public health effort (such as publicizing vaccination and media coverage of infection) under voluntary vaccination and incorporating human vaccination behaviour during an influenza outbreak remain elusive. A recent work by the same authors, in simpler framework, incorporates human decision-making in the optimal budget allocations for controlling infectious disease (Deka and Bhattacharyya 2019).

In this paper, we consider a framework combining compartmental SIR model for disease dynamics and evolutionary game theory to describe human decision-making under voluntary vaccination during a flu outbreak. To understand the impact of timedependent public health effort on minimizing the disease burden in the population, we also incorporate the effect of awareness campaign by public health agency in the utility of vaccination game using different functional forms. Applying Pontryagin's maximum principle (PMP) on the prevalence-behaviour model, we observe that under different functional forms, model exhibits qualitatively and quantitatively different patterns in the epidemic outbreak and optimal cost due to public health effort. The dynamics of vaccination and disease intimately depends on individual risk perceptions that influenced by information such as current vaccine coverage or disease induced death. A numerical simulation of the model also highlights that optimal cost from infection is much higher when there is a delay in initiating the control effort in the beginning. On the contrary, policymakers may reduce the allocation of budgets during the peak of the outbreak or when there is high death or hospitalization rate, and yet the disease burden will not escalate. The perceived risk of vaccine and infection evolves over time, and therefore, understanding the strategic interplay between individual behaviour and disease dynamics informed by perceived vaccine and disease costs during such voluntary involvement may guide policymakers to reduce the cost and their effort in controlling a disease outbreak.

The article is framed as follows: In Sect. 2, we describe the game-theoretic model of vaccination dynamics and integrate with compartmental disease prevalence model. We also define public health effort and develop optimal control problem. In Sect. 3, we analyse our optimization problem using Pontryagin's maximum principle (PMP) and deduce the optimal solution. In Sect. 4, we analyse our model with varying perceived risk of vaccination and disease deaths using PMP. In Sect. 5, we present our numerical results through simulations. Finally, in Sect. 6, we conclude our findings with limitations.

\section{Model and Assumptions}

For disease transmission, we consider a homogenous well-mixed population. For simplification, we assume that there is no birth and death (natural) in populations. (Influenza outbreak lasts for half a year or so.) The immunity of the vaccine lasts even less than a year, and there is no control measure other than vaccination. We further assume that the vaccine is new, and hence, individuals' perceptions about the vaccine risks are much higher at the beginning of the outbreak. 


\subsection{Disease Prevalence Model}

We consider compartmental SIR model for the disease transmission, where the total population is divided based on the disease status as susceptible $(S)$, infected $(I)$ and recovered $(R)$ (Anderson and May 1992). We can write the combined system of equation as

$$
\begin{aligned}
\frac{\mathrm{d} S}{\mathrm{~d} t} & =-\beta S I \\
\frac{\mathrm{d} I}{\mathrm{~d} t} & =\beta S I-\gamma I \\
\frac{\mathrm{d} R}{\mathrm{~d} t} & =\gamma I
\end{aligned}
$$

The parameter $\beta$ is the mean transmission rate of the infection, and $1 / \gamma$ is the mean infectious period of the population. In this framework, we assume the population is homogeneous, strongly mixed. It is closed population, and the duration of infections is exponentially distributed. The initial condition is $S(0) \sim 0.99$ and $I(0) \sim 0.01$.

\subsection{Vaccination Decision: Game-Theoretic Model}

In our vaccination population game, individuals' perception about vaccination evolves with time depending on disease prevalence. We model the vaccination decision as twostrategy pairwise contest game (Hofbauer and Sigmund 1998).

In this game, we define the population state as $\mathbf{x}(t)=\left(x_{1}(t), x_{2}(t)\right)$, and let $S_{n}=$ $\{V, \mathrm{NV}\}$ be the two pure strategies of the population. Suppose $x_{1}(t)$ is the fraction of population using strategy $V$ (vaccination) per unit time (day) and $x_{2}(t)=1-x_{1}$ is the fraction of population using strategy NV (non-vaccination) per unit time. Following the derivation given in Hofbauer and Sigmund (1998), we have the growth equation of the vaccinator population as:

$$
\dot{x}=x(1-x)(\pi(V, \mathbf{x})-\pi(\mathrm{NV}, \mathbf{x}))
$$

Similarly for $x_{2}=1-x$, i.e. individuals who switch from vaccinator to non-vaccinator strategy, we will arrive at the same Eq. (2). This is same as the replicator equation in the evolutionary game theory (Hofbauer and Sigmund 1998).

Under voluntary vaccination campaign during an outbreak such as influenza, it was observed that individuals perceptions are related to the safety of the vaccine, socioeconomic and religious view and also the perceived probability of suffering significant morbidity upon infection (Rogers et al. 2018). Thus, the fitness cost of vaccination or non-vaccination depends on such vaccine safety, and risk of morbidity upon infection. Individuals also tend to reap the benefits of herd immunity and therefore try to evade vaccination when the vaccine coverage is about the social optimum and goes for vaccination more readily when coverage is critically low (Bauch and Bhattacharyya 2012). 
We consider the perceived pay-off for adopting vaccinator strategy is

$$
\pi(V, \mathbf{x})=-r_{\mathrm{v}},
$$

where $r_{v}$ is the probability of suffering significant morbidity from the vaccine.

The perceived pay-off for adopting non-vaccinator strategy is

$$
\pi(\mathrm{NV}, \mathbf{x})=-r_{\mathrm{i}} m \times \text { disease status }
$$

where $r_{\mathrm{i}}$ is the probability of suffering significant morbidity upon infection, and parameter $m$ quantifies the sensitivity of vaccinating behaviour that tends to change with the disease status. Individual may depend on current disease prevalence $I(t)$ or daily new infections $\beta S(t) I(t)$ for perceiving the disease status. We discuss both cases in this paper, and the results of the model with daily new infections $\beta S(t) I(t)$ are in Supplementary Information (Sect. S2).

However, the pay-off matrix, when individual would interact with other members in the population and calculate the pay-off for other strategy, is as follows:

\begin{tabular}{lll}
\hline & Player $Y$ & \\
\hline & $V$ & $\mathrm{NV}$ \\
\hline Player $X$ & & \\
$V$ & $\left(-r_{\mathrm{V}},-r_{\mathrm{V}}\right)$ & $\left(-r_{\mathrm{V}},-r_{\mathrm{i}} m I\right)$ \\
$\mathrm{NV}$ & $\left(-r_{\mathrm{i}} m I,-r_{\mathrm{V}}\right)$ & $\left(-r_{\mathrm{i}} m I,-r_{\mathrm{i}} m I\right)$ \\
\hline
\end{tabular}

Thus, the evolutionary equation of frequency of vaccination strategy is given by

$$
\dot{x}=x(1-x)\left(-r_{\mathrm{v}}+r_{\mathrm{i}} m I(t)\right) .
$$

We can define the expression $\Delta E:=-r_{\mathrm{v}}+r_{\mathrm{i}} m I$ as the pay-off gain to switch one strategy to the other. Note that individuals would tend to switch their strategy, provided other members of the population who have adopted a different strategy get a higher pay-off. If $\Delta E>0$, a non-vaccinator changes its strategy to a vaccinator strategy, and when $\Delta E<0$, a vaccinator chooses not to vaccinate, and thus, frequency of vaccinators decreases (Fig. 1).

This equation may be interpreted as imitation dynamics in vaccination decision. It may be assumed that individuals randomly sample other members of the population during the decision and imitate their strategies provided it gives them a higher pay-off, where the parameter $k$ is the sampling rate (Bauch 2005; Reluga et al. 2006).

However, Eq. (5) is the same as replicator equation in evolutionary game theory (Hofbauer and Sigmund 1998). 
Fig. 1 Schematic of switching between strategies where $\Delta E$ is the pay-off gain for adopting strategies vaccination and non-vaccination

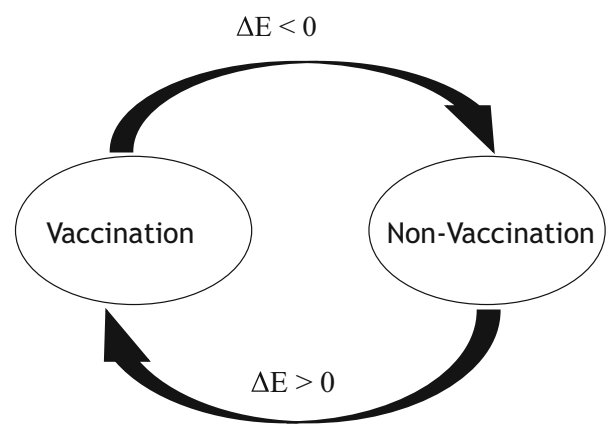

\subsection{Public Health Effort and Vaccinating Decision}

Public health ministries initiate vaccine campaign, media awareness on vaccine safety and availability, forecasting disease prevalence, etc., for controlling the outbreak. They have a limited budget which they disburse over the period of the entire outbreak. This public health effort works as an incentive for individual vaccinations. In our model, we consider $u(t) \in\left[0, u_{\max }\right]$ is the public health initiative during the course of the outbreak, and $f(u(t))$ denotes the change in public perceptions considering this as an incentive for vaccination. This, however, impacts the pay-off gain of individual decision in vaccination. As we have no empirical support how such public health initiative impacts public perceptions, we analyse the model system assuming $f$ has different functional forms like linear, exponential, quadratic, hyperbolic, and square root. Coupling this incentive from the policymakers, the new pay-off for individuals would be

$$
-r_{\mathrm{V}}+r_{\mathrm{i}} m I+f(u(t))
$$

We integrate the disease model and vaccination decision model including the effect of public health effort to get the final model:

$$
\begin{aligned}
\frac{\mathrm{d} S}{\mathrm{~d} t} & =-\beta S I-x S \\
\frac{\mathrm{d} I}{\mathrm{~d} t} & =\beta S I-\gamma I \\
\frac{\mathrm{d} R}{\mathrm{~d} t} & =\gamma I \\
\frac{\mathrm{d} x}{\mathrm{~d} t} & =k x(1-x)\left(-r_{\mathrm{v}}+r_{\mathrm{i}} m I+f(u)\right)
\end{aligned}
$$

with similar assumptions and initial conditions like the basic model (1), with $x(0)=$ $x_{0}>0$, as there is always some advance vaccinators in any real population. This type of behaviour-prevalence model had also been introduced as special case earlier (Bauch 2005; Bauch and Bhattacharyya 2012; Deka and Bhattacharyya 2019). 


\section{Formulation of Our Objective Function}

Our objective is to reduce the number of new infections through vaccination and also the cost due to public health effort. New incidence is always a better measure than disease prevalence from public health perspective. The formulation of our objective function which is to be maximized over a set of control parameters is defined as follows:

$$
J(u)=\int_{0}^{T}\left[-c_{\mathrm{I}} \beta S(t) I(t)-\eta u(t)\right] \mathrm{d} t,
$$

where $\eta$ is the cost of per unit public health initiative $u(t)$ and $c_{\mathrm{I}}$ is the cost of infection per new infective. $(S, I, R, x)(0)=\left(S_{0}, I_{0}, R_{0}, x_{0}\right)$ and $T=\inf \{t \mid I(t)$ is near zero or infection dies out $\}$. As discussed above, we have also the constraint $\int_{0}^{T} u(t) \mathrm{d} t \leq$ $T u_{\max }$.

It may be worthwhile to discuss here how we choose $T$, the terminal time in the optimal control. Theoretically, the end of the epidemic in such models system (7) is not well defined, because the number of infected individuals approaches zero asymptotically as time approaches infinity. So, it is not immediately clear how best to choose $T$, especially for practical purpose. Hence, the three usual options are available: (i) $T=\infty$, (ii) $T=T_{\max }$, where $T_{\max }$ is sufficiently large constant, and (iii) $T=\inf \left\{t \mid I(t)=I_{\min }\right\}$, where $I_{\min }$ is such a minimum values of infection that can be assumed to indicate the end of epidemic.

In case of (i), there may have potential problem to occur at the very large values of $t$ by the model system, which may further impact the optimal control problem. For example, suppose $u(t)$ is nonzero, and then, at some large values of $t=t_{1}, I\left(t_{1}\right)<<1$. As it satisfies the extra constraint on $u(t): \int_{0}^{T} u(t) d t \leq T u_{\max }, u(t)$ may be 0 for all $t>t_{1}$. In such case, if $\dot{I}(t)>0 \forall t>t_{1}$, however, then there will be another peak of infective before the epidemic dies out completely. We can avoid such unusual behaviour by considering $T$ is finite. Also for practical purpose, it is more acceptable in optimal control that $T$ is finite. In case of (ii), it is not clear how large we should take $T$ in order to coincide with a reasonable interpretation of the end of the epidemic. Option (iii) $I(t)=I_{\text {min }}$ avoids both of these difficulties, because manually chosen $I_{\min }$ indicates the termination of the epidemic in a finite time. In our simulation, we have chosen $I_{\min }$ a very low value, which reflects one flu outbreak in a year.

\subsection{The Optimal Control Problem}

We analyse the behaviour of our model in Eq. (7) by using the optimal control theory for different choices of $f(u)$. We seek the optimal control $u^{*}$ for our objective function given in Eq. (8) such that

$$
J\left(u^{*}\right)=\max \{J(u): u \in \Omega\}
$$

where $\Omega$ is the control set defined by

$$
\Omega=\left\{u: 0 \leq u(t) \leq u_{\max }, t \in[0, T]\right\}
$$




\subsection{Solution of the General Problem}

Theorem 1 (a) There exist optimal control $u^{*} \in \Omega$ and solutions of $S^{*}, I^{*}, x^{*}$ such that $J\left(u^{*}\right)=\max J(u)$ over $[0, T]$. Further there exist adjoint variables $\lambda_{S}, \lambda_{I}$, $\lambda_{x}$ satisfying the adjoint equation

$$
\frac{\mathrm{d} \lambda_{i}}{\mathrm{~d} t}=-\frac{\partial H\left(t, x^{*}, u^{*}, \lambda\right)}{\partial i}, \quad i=S, I, x
$$

with transversality condition $\lambda_{S}^{*}(T)=\lambda_{I}^{*}(T)=\lambda_{x}^{*}(T)=1$.

(b) The optimal control $u^{*}$ which maximizes $J$ over the region $\Omega$ is given by $u^{*}(t)=$ $\min \left(u_{k}^{*}, u_{\max }\right)$ where $u_{k}^{*}$ is obtained from equation

$$
f^{\prime}\left(u_{k}^{*}\right)=\frac{\eta}{\lambda_{x} k x^{*}\left(1-x^{*}\right)}
$$

Proof We use the help of Lenhart et al. (2007) to prove the existence of the theorem.

(a) The integrand $J(u)=-c_{\mathrm{I}} \beta S(t) I(t)-\eta u(t)$ is convex with respect to $u(t)$ on the control set $\Omega$.

(b) The state system is Lipschitz with respect to the state variables, and also, the control variable $u \in \Omega$ is also closed and bounded by the definition.

Therefore, there exists an optimal control for minimizing the objective functional subject to the constraint. We apply Pontryagin's maximum principle to derive the necessary conditions to find the optimal solutions as follows:

We construct the Hamiltonian from the disease dynamics and the objective function

$$
H((S, I, x)(t), u(t), \lambda(t), t)=-c_{I} \beta S I-\eta u(t)+\lambda_{S} A+\lambda_{I} B+\lambda_{x} C
$$

where

$$
\begin{aligned}
& A=-\beta S I-x S \\
& B=\beta S I-\gamma I \\
& C=k x(1-x)\left(-r_{\mathrm{v}}+r_{\mathrm{i}} m I+f(u)\right)
\end{aligned}
$$

$\lambda_{S}, \lambda_{I}, \lambda_{x}$ are the associated adjoint variables for the states $S, I, x$, respectively.

The adjoint equations evaluated at the optimum are given by

$$
\begin{aligned}
& \lambda_{S}^{\prime}(t)=-\frac{\partial H}{\partial S}=c_{\mathrm{I}} \beta I^{*}+\lambda_{S}\left(\beta I^{*}+x^{*}\right)-\lambda_{I} \beta I^{*} \\
& \lambda_{I}^{\prime}(t)=-\frac{\partial H}{\partial I}=c_{\mathrm{I}} \beta S^{*}+\lambda_{S} \beta S^{*}-\lambda_{I}(t)\left(\beta S^{*}-\gamma\right)-\lambda_{x} k x^{*}\left(1-x^{*}\right) r_{\mathrm{i}} m \\
& \lambda_{x}^{\prime}(t)=-\frac{\partial H}{\partial x}=\lambda_{S} S^{*}-\lambda_{x}\left[k\left(1-2 x^{*}\right)\left(-r_{\mathrm{v}}+r_{\mathrm{i}} m I^{*}+f\left(u^{*}\right)\right)\right]
\end{aligned}
$$

(b) We use the Pontryagin maximum principle to differentiate the Hamiltonian $H$ with respect to $u$ on the set $\Omega$ : 


$$
\frac{\partial H}{\partial u}=0
$$

upon simplifying which gives

$$
f^{\prime}\left(u_{k}^{*}\right)=\frac{\eta}{\lambda_{x} k x^{*}\left(1-x^{*}\right)} .
$$

Remark 1 This, however, gives different forms of $u_{k}^{*}$, when different forms of function $f$ are chosen:

(i) Square root: $f(u)=\xi \sqrt{u(t)}$ then $\sqrt{u_{k}^{*}}=\frac{\xi \lambda_{x} k x^{*}\left(1-x^{*}\right)}{2 \eta}$

(ii) Hyperbolic: $f(u)=\frac{\xi u(t)}{b+c u(t)}$, then $u_{k}^{*}=\frac{\xi \sqrt{\lambda_{x} k x^{*}\left(1-x^{*}\right) b \xi}}{c \sqrt{\eta}}-b$

(iii) Quadratic: $f(u)=\xi u^{2}(t)$, then $u_{k}^{*}=\frac{\eta}{2 \xi \lambda_{x} k x^{*}\left(1-x^{*}\right)}$

(iv) Exponential: $f(u)=e^{\xi u(t)}$, then $u_{k}^{*}=\frac{1}{\xi} \log \left(\frac{\eta}{\xi \lambda_{x} k x^{*}\left(1-x^{*}\right)}\right)$

When $f(u)$ is linear in $u$, the control is Bang-Bang, and we separately present this case in Supplementary Information.

\section{Optimal Public Health Effort in Varying Perceived Risk of Vaccination and Disease Deaths}

Individuals risk perception about vaccines is much higher in the beginning, as in most cases, the vaccines are new (Gerdil 2003; Carrat and Flahault 2007). However, the perception declines among the population as more individuals vaccinate, and the vaccine safety information spreads in the population. More and more people become aware of the misperception of the adverse effects of vaccine risks through the word of mouth, more they choose to vaccinate. On the other hand, increased deaths and hospitalizations due to severity from infection also motivate individuals for vaccination (Louie et al. 2009; Viboud et al. 2006). This is due to the fact that the pay-off of vaccinator strategy becomes higher than the non-vaccinator strategy and herd immunity level has not been attained in the community.

Here, we assume that the perception of the vaccine risks declines exponentially with increasing vaccine coverage $V_{d}$, and the pay-off for vaccination increases linearly with increasing deaths. Incorporating this pay-off in our model of Eq. (7) with $f(u)=\xi u$, we arrive at

$$
\begin{aligned}
\frac{\mathrm{d} S}{\mathrm{~d} t} & =-\beta S I-x S \\
\frac{\mathrm{d} I}{\mathrm{~d} t} & =\beta S I-\gamma I-\mathrm{d} I \\
\frac{\mathrm{d} x}{\mathrm{~d} t} & =k x(1-x)\left(-r_{\mathrm{v}} e^{-\delta V_{d}}+a d I+r_{\mathrm{i}} m I+\xi u\right) \\
\frac{\mathrm{d} V_{d}}{\mathrm{~d} t} & =x S
\end{aligned}
$$


$V_{d}$ is the compartment of vaccine uptake. The parameters $\delta$ and $a$ are sensitivity parameters in response to vaccination and death, respectively. We also consider the objective function $J(u)$ is quadratic in $u$, i.e.

$$
J(u)=\int_{0}^{T}\left[-c_{\mathrm{I}} \beta S(t) I(t)-\eta u^{2}(t)\right] \mathrm{d} t,
$$

Theorem 2 For optimal public health effort with varying perceived risk of vaccination, there exist optimal control $u^{*} \in \Omega$ and solutions of $S^{*}, I^{*}, x^{*}$ and $V_{d}^{*}$ from model (13) such that $J\left(u^{*}\right)=\max J(u)$ over $[0, T]$. Further there exist adjoint variables $\lambda_{S}$, $\lambda_{I}, \lambda_{x}$ and $\lambda_{V_{d}}$ satisfying the adjoint equation

$$
\frac{\mathrm{d} \lambda_{i}}{\mathrm{~d} t}=-\frac{\partial H\left(t, x^{*}, u^{*}, \lambda\right)}{\partial i}, \quad i=S, I, x \text { and } V_{d}
$$

with transversality condition $\lambda_{S}^{*}(T)=\lambda_{I}^{*}(T)=\lambda_{x}^{*}(T)=\lambda_{V_{d}}^{*}(T)=1$.

(b) The optimal control $u^{*}$ which maximizes $J$ over the region $\Omega$ is given by $u^{*}=$ $\min \left\{u_{k}^{*}, u_{\max }\right\}$ where $u_{k}^{*}$ is defined by

$$
u_{k}^{*}=\frac{\xi \lambda_{x} k x^{*}\left(1-x^{*}\right)}{2 \eta} .
$$

Proof (a) The proof is similar to Theorem (1). The Hamiltonian with model (13) is as follows,

$$
H((S, I, x)(t), u(t), \lambda(t), t)=-c_{\mathrm{I}} \beta S I-\eta u(t)^{2}+\lambda_{S} A+\lambda_{I} B+\lambda_{x} C+\lambda_{V_{d}} D,
$$

where

$$
\begin{aligned}
& A=-\beta S I-x S \\
& B=\beta S I-\gamma I-d I \\
& C=k x(1-x)\left(-r_{\mathrm{v}} e^{-\delta V_{d}}+a d I+r_{\mathrm{i}} m I+f u(t)\right) \\
& D=x S
\end{aligned}
$$

$\lambda_{S}, \lambda_{I}, \lambda_{x}, \lambda_{V_{d}}$ are the associated adjoint variables for the states $S, I, x, V_{d}$, respectively. The adjoint equations evaluated at the optimum are given by

$$
\begin{aligned}
\lambda_{S}^{\prime}(t)= & -\frac{\partial H}{\partial S}=c_{\mathrm{I}} \beta I^{*}+\lambda_{S}\left(\beta I^{*}+x^{*}\right)-\lambda_{I} \beta I^{*}-\lambda_{V_{d}} x^{*} \\
\lambda_{I}^{\prime}(t)= & -\frac{\partial H}{\partial I}=c_{\mathrm{I}} \beta S^{*}+\lambda_{S} \beta S^{*}-\lambda_{I}(t)\left(\beta S^{*}-\gamma-d\right) \\
& -\lambda_{x} k x^{*}\left(1-x^{*}\right)\left(r_{\mathrm{i}} m+a d\right) \\
\lambda_{x}^{\prime}(t)= & -\frac{\partial H}{\partial x}=\lambda_{S} S^{*}-\lambda_{x}\left[k ( 1 - 2 x ^ { * } ) \left(-r_{\mathrm{v}} e^{-\delta V_{d}}\right.\right.
\end{aligned}
$$




$$
\begin{array}{r}
\left.\left.+a d I^{*}+r_{\mathrm{i}} m I^{*}+f\left(u^{*}\right)\right)\right]-\lambda_{V} S^{*} \\
\lambda_{V_{d}}^{\prime}(t)=-\frac{\partial H}{\partial V_{d}}=-\lambda_{x} k x^{*}\left(1-x^{*}\right) r_{\mathrm{v}} \delta e^{-\delta V_{d}}
\end{array}
$$

(b) The proof is similar to Theorem (1).

Remark 2 Note that there is no empirical support that how individual perceptions change with available vaccine safety information or death due to severity from infection. Here, we explored cases with exponential functions for vaccination risk and linear with disease death. However, we also analyse the situation when both are linear functions and details are given in Supplementary Information (Fig. S5).

\section{Numerical Results}

We numerically simulate the model Eq. (7) and adjoint Eq. (12) using forwardbackward sweep iterative method with a fourth-order Runge-Kutta scheme to obtain the optimal solution $\left(S^{*}, I^{*}, x^{*}\right)$ and $u^{*}$ in different situations discussed in earlier sections. Starting with initial guesses for the controls, the state equations are solved forward in time. Then, those state values are used to solve the adjoint equations backward in time. The controls are updated, and the process is repeated until convergence is reached (Lenhart et al. 2007). We have calibrated the parameters to replicate flu-like diseases and scaled $u_{\max }$ to 1 for all simulations in this study. The parameter range is provided in Table 1.

\subsection{Human Vaccinating Behaviour Conserves the Timing and Size of Peak of the Outbreak}

It is observed in a compartmental epidemic model that the peak of the outbreak changes with an increase in transmissibility of infection. For example, the peak size increases and peak timing shifts towards left with the increase in basic reproduction ratio $\left(R_{0}\right)$. Figure 2 a depicts an increase in basic reproduction ratio $R_{0}$ from 2 to 4 , the peak of the outbreak moves towards the left (200 to 100 days). In contrast, if we incorporate behavioural aspect of individuals in the model, it is observed that the peak of the outbreak is conserved even for different values of basic reproduction ratio $R_{0}$ from 2 to 4 . Figure $2 b$ illustrates that the peak of the outbreak is within the same time interval, i.e. first 30 days. This can be attributed to individuals risk perceptions and vaccination decision. Advance and early vaccination becomes more common as $R_{0}$ increases. Individuals experience a higher force of infection with increased $R_{0}$ and hence will seek vaccination early during the onset of the outbreak. This works as a feedback mechanism to keep the peak timing unchanged: higher $R_{0}$ stimulates more individuals to adopt advance or early vaccination to fend off infection, which, in turn, counteracts the effect of higher $R_{0}$ by delaying the epidemic peak. 


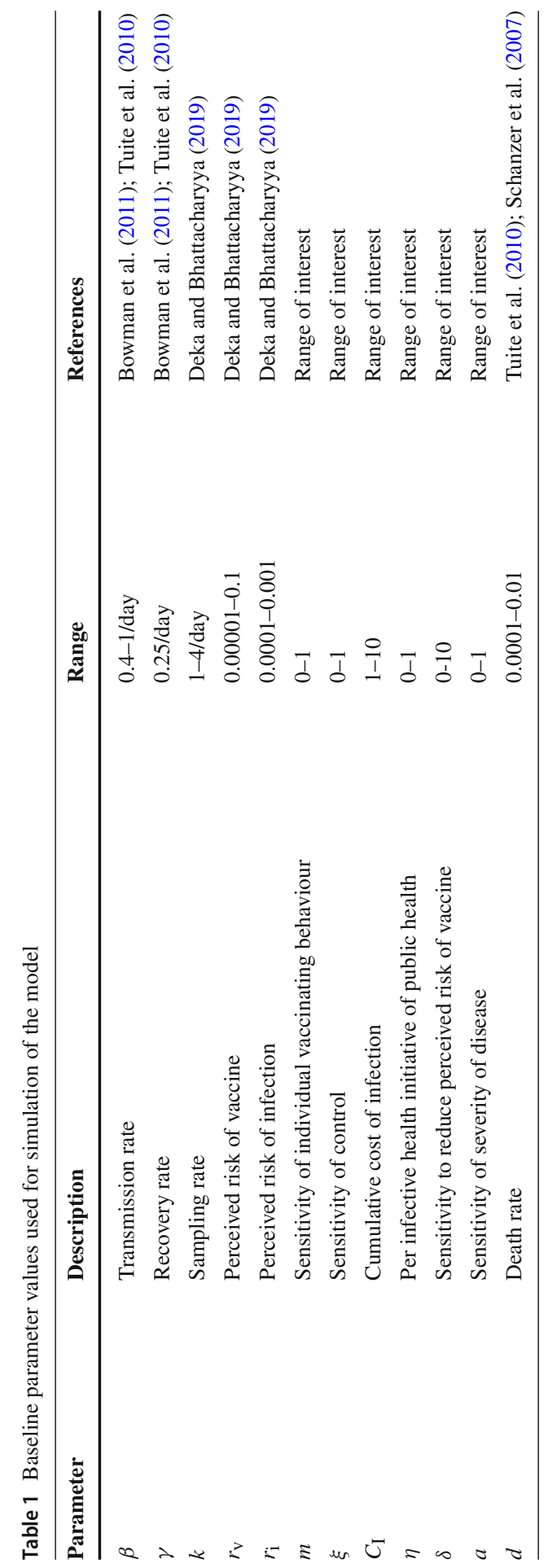


(A)

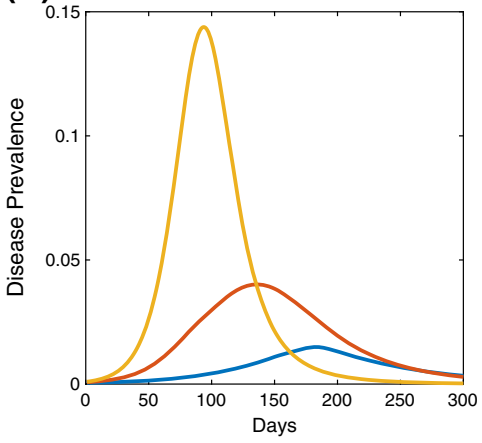

(B)

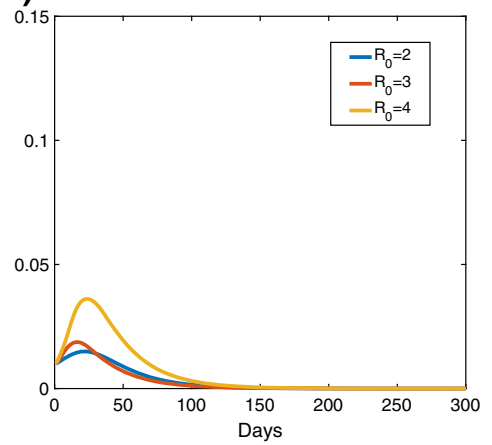

Fig. 2 Timing of outbreak for an influenza model with respect to varying disease transmissibility a without vaccination behaviour, $\mathbf{b}$ with individual vaccinating decision included. Here, we have consider $u=0$, and the parameter values are defined in Table 1. The results highlight that the peak of the outbreak is conserved when we consider the human vaccinating behaviour

\subsection{Optimal Public Health Effort and Vaccination}

Public perceptions and sentiments towards awareness program, vaccination campaign or healthcare service, especially, during an epidemic play a major role behind the success story of disease management strategies. Here, we have used different functional forms to simulate the model that how public health effort during an epidemic may act as an incentive for individual decision-making in vaccination. Among four different incentive functions - exponential, quadratic, hyperbolic and square root-we see that exponential and quadratic are more effective, as the peak size is lowest, vaccine uptake is higher, and the underlined optimal cost is minimum (Fig. 3). In contrast, hyperbolic and square root functional forms exhibit less impact on reducing the infection, and optimal cost is also very high compared to the other two functional forms. The linear function defines Bang-Bang control, which we discuss in Supplementary Information (Sect. S1). Thus, different forms of public perceptions and attitudes towards healthcare service may bring interesting qualitative difference in the dynamics of disease outbreak and may incur different optimal public health costs in disease management. To experiment more on these qualitative and quantitative differences and their dependence on the sensitivity parameter $\xi$ and disease transmission parameter $R_{0}$, we simulate the model Eq. (7) and plot the cumulative incidence, optimal control, total cost and daily proportion of vaccinators under different values of $\xi$, and $R_{0}$. Higher $\xi$ defines that the individuals are more sensitive towards public health measures.

Figure 4 shows the effect of variations in $R_{0}$ and the sensitivity parameter $\xi$. Higher $R_{0}$ increases the cumulative infection, which increases the optimal public health effort and cumulative cost for controlling the disease. The interesting observation is that the daily proportion of vaccinators $(x)$ increases only when there is enough sensitivity from the public health officials. As the parameter $\xi$ increases, the daily proportion of vaccinators also increases. The results highlight that policymakers should be very sensitive and give optimum effort to inform the public about the severity of the disease, when the actual risk is very high. Here, we demonstrate the plot (Fig. 4) with the 

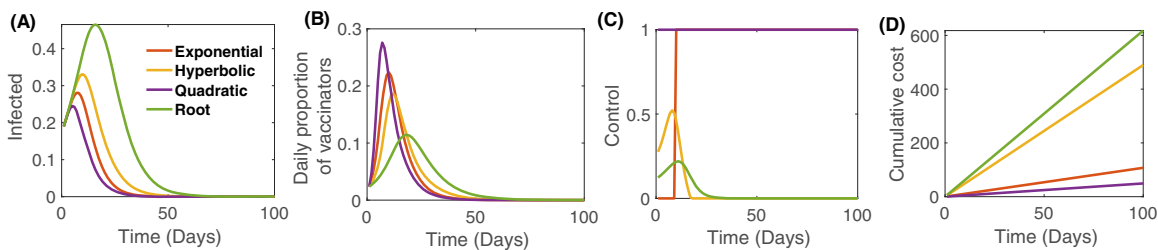

Fig. 3 Disease dynamics under different control functions (exponential, hyperbolic, quadratic and square root): a Infectives, $\mathbf{b}$ daily proportion of vaccinators, $\mathbf{c}$ cumulative public health effort, $\mathbf{d}$ cumulative cost (absolute value) due to infection and control. Parameter regimes are $k=9, C I=1, \eta=0.95$, and other parameter values are defined in Table 1

(A)

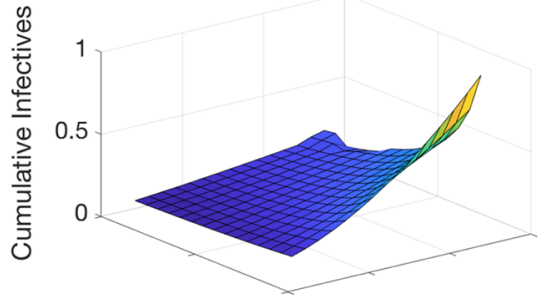

(C)

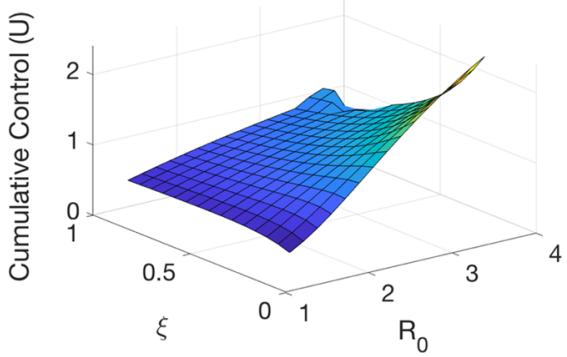

(B)

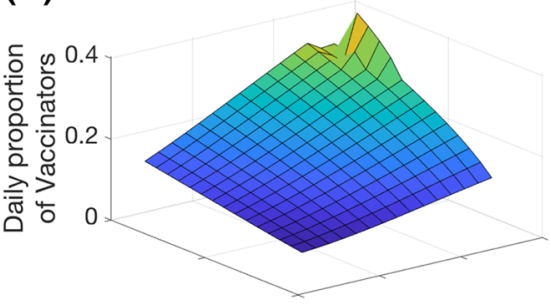

(D)

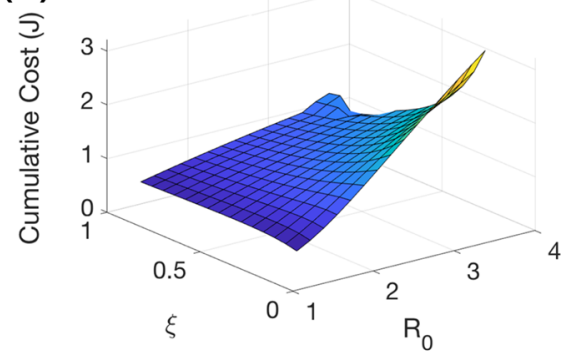

Fig. 4 Dynamics of disease and vaccination of the optimal control model (7) under different values of $R_{0}$ and $\xi$ (sensitivity of public health towards disease prevalence). The control function is taken as hyperbolic: $f(u)=\xi u(t) /(b+c u(t))$. a Cumulative infective, $\mathbf{b}$ daily proportion of vaccinators, $\mathbf{c}$ cumulative effort from public health and $\mathbf{d}$ cumulative cost (absolute value). Here, the parameter regimes are $\eta=0.95$, $r_{\mathrm{V}}=0.00001, r_{\mathrm{I}}=0.01, k=4$. Other parameter values are defined in Table 1

hyperbolic functional form of public perceptions, and analysis for other forms is given in Supplementary Information (Figs. S2-S4).

\subsection{Optimal Effort with Delay and Gap During Outbreak}

The serotype of influenza strain changes every year through antigenic drift, and hence, in most of cases, the vaccine does not work for the next year (Liu et al. 2012). Individuals have almost no immunity from current year's vaccine against the new strain that may emerge in the next year. This, however, increases the transmission of pathogen in the beginning, which makes a serious challenge to public health officials for the 
Control with delay

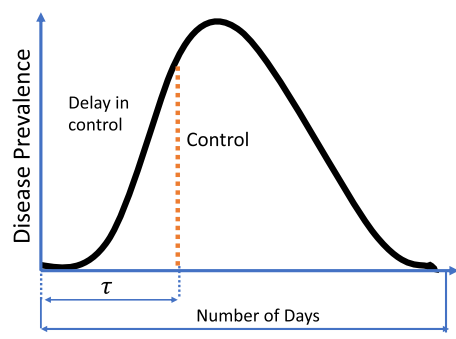

Control with gap

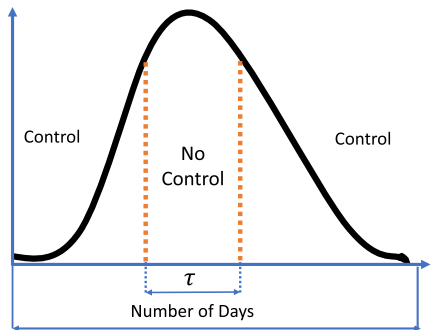

Fig. 5 Schematic diagram of public health effort with number of days in delay and gap. The parameter $\tau$ is the period of no effort from public health

timely delivery of vaccines. In the past, even developed nations are unable to provide sufficient vaccine to tackle the outbreak such as 2004-2005 influenza vaccine shortage in the USA (Engler et al. 2008; Ferguson et al. 2006).

We assume that every year a new influenza vaccine is developed and individuals have no idea about the side effects of the vaccine which makes their perception about the vaccine risk much higher in the beginning, and so, they avoid getting vaccinated (Brewer and Hallman 2006). From the game-theoretic perspective, in such cases, delaying vaccination can be the best strategy as individuals can reap the benefit of herd immunity provided by the early vaccinators. But, if everyone tends to delay vaccination at the beginning, ultimately the herd immunity level is never achieved. When no individual goes for vaccination, the disease prevalence rapidly increases and an outbreak emerges. On the other hand, when the infection reaches its peak, individuals may tend to go for vaccination, because it gives a higher pay-off as opposed to the non-vaccinator strategy. At the high infection rate, there will have minimal impact of vaccination campaign, economic and social issues on increasing the vaccine coverage.

Here, we explore how rapid or late deployment of public health interventions during an outbreak may impact the voluntary vaccination dynamics and disease prevalence. Policymakers may have limited resources at its disposal to control infections. They may employ two policies - they will wait and employ control only when the outbreak reaches a certain level or they might provide control only at the beginning or late of the outbreak when individual initiative for vaccination is less due to low disease prevalence.

To test the hypothesis, we implement this both on behavioural model (7) and a nonbehavioural model of vaccination and disease transmission. In the non-behavioural model, we assume that vaccine uptake rate proportionately increases with the public health effort $u(t)$ irrespective of disease prevalence. We consider two scenarios in each case: (1) Fig. 6, where the policymakers delay from 1 to 20 days in providing the effort in the beginning, whereas (2) Fig. 7, where the policymakers do not initiate any effort for even number of days ranging from 2-20 days during the peak of outbreak, (1-10 days on either side of the peak of the outbreak). Our analysis shows that under a gametheoretic approach, policymakers should be proactive at the beginning of an outbreak. 
(A)
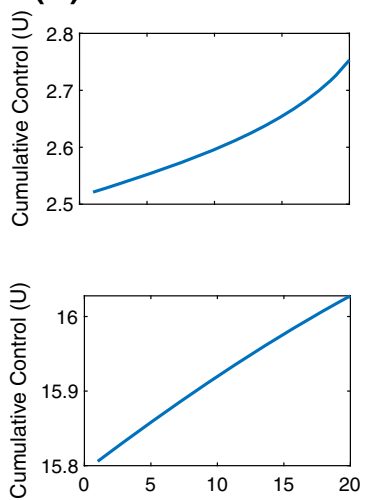

(B)

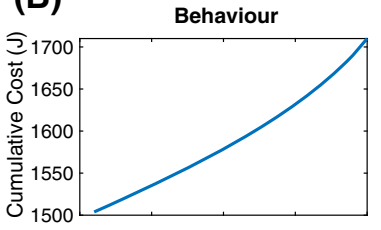

Non-Behaviour

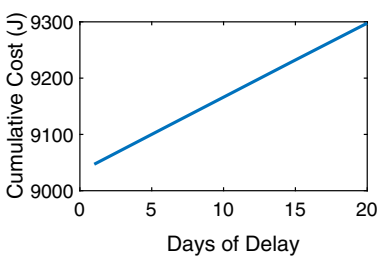

(C)
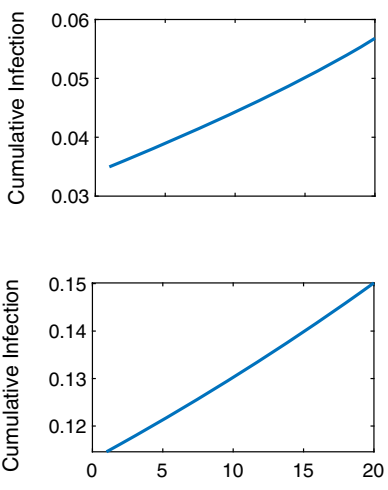

Fig. 6 Analysis of our model with initial delay in release of effort from public health. Delay from the public health could be due to unavailability of vaccine at the onset of the outbreak or due to insensitivity owing to low disease prevalence (see Fig. 5 for reference). The control function for behaviour model is taken as square root. a Cumulative control, b cumulative cost (absolute value), c cumulative infection. Here, the parameter regimes are $R_{0}=4, r_{\mathrm{V}}=0.00001, r_{\mathrm{I}}=0.01, k=7, \xi=0.9, \eta=0.95$. Other parameter values are defined in Table 1

(A)
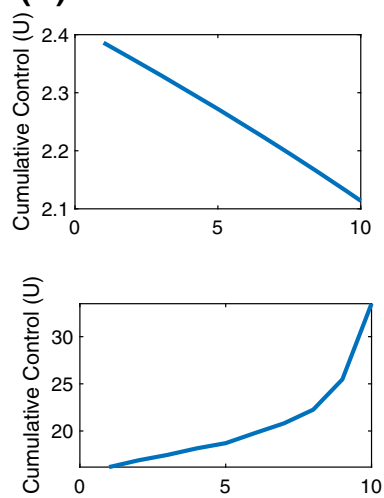

(B)

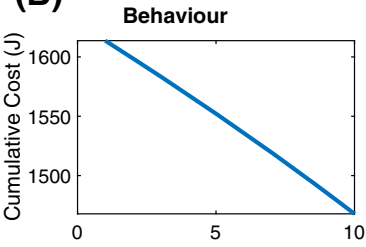

Non-Behaviour

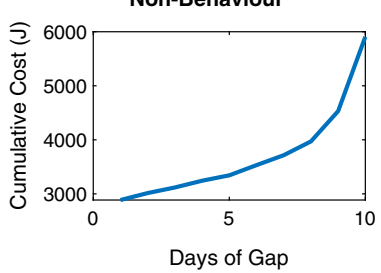

(C)
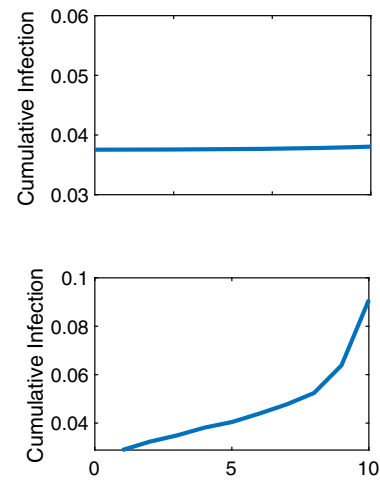

Fig. 7 Analysis of our model with gap in release of effort from public health. There is no public health effort implemented during the gap (see Fig. 5 for reference). The control function for behaviour model is taken as square root. a Cumulative control, b cumulative cost (absolute value), c cumulative infection. Here, the parameter regimes are $R_{0}=4, r_{\mathrm{V}}=0.00001, r_{\mathrm{I}}=0.01, k=7, \xi=0.9, \eta=0.95$. Other parameter values are defined in Table 1

From Fig. $6 \mathrm{c}$ with initial delay, the cumulative incidence increases rapidly (from 0.37 to 0.57 ), whereas from Fig. $7 \mathrm{c}$ with a gap during the peak of the outbreak, there is almost no increase in the disease prevalence. The interesting observation is that the optimal effort and cumulative cost for disease management increase as the initial delay increases from the public health (Fig. 6a and b). However, the scenario is opposite in case of public health effort with gap-both optimal effort and total cumulative cost 
(A)

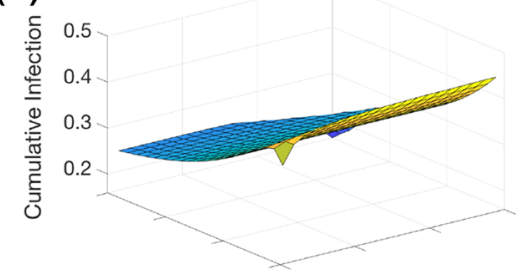

(C)

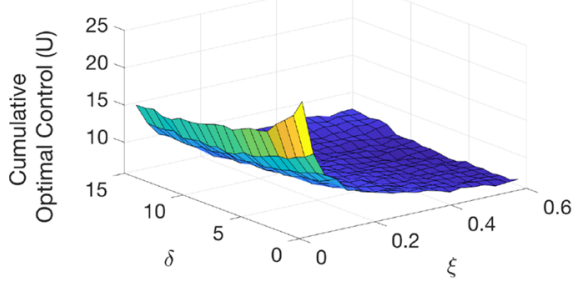

(B)

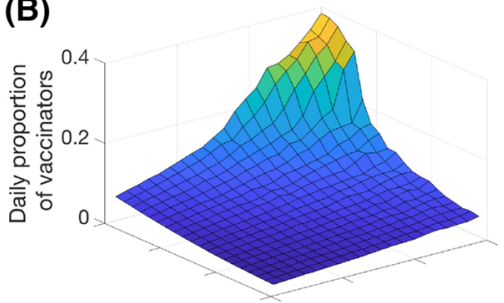

(D)

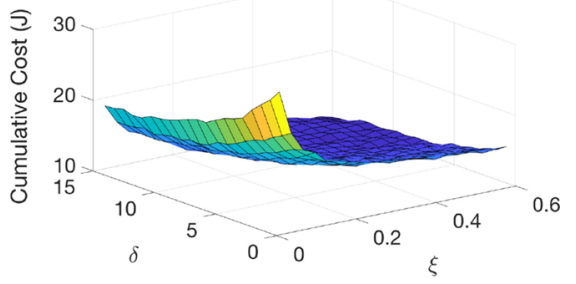

Fig. 8 Analysis of the model (13) where the perceived risk of vaccine declines with increased uptake. Here, $\delta(0-15)$ is the sensitivity to decline perceived risk of vaccine and $\xi$ is the sensitivity parameter for control of public health. Higher $\delta$ indicates that the perception of the vaccine risk is declining exponentially. Higher $\xi(0-0.6)$ implies that public health effort is more proactive. The control function for behaviour model is taken as square root. a Cumulative infective, $\mathbf{b}$ daily proportion of vaccinators, $\mathbf{c}$ cumulative public health effort, $\mathbf{d}$ cumulative cost. Here, the parameter regimes are $R_{0}=3, r_{\mathrm{V}}=0.02, r_{\mathrm{I}}=0.05, k=4, \eta=0.95$.

Other parameter values are defined in Table 1

decreases significantly (Fig. 7a and b). This difference in the dynamics observed as the initiation of public health effort, in the beginning, increases the daily vaccination level in such a high level. The high vaccination uptake, in the beginning, feeds back on the disease prevalence and reduces the incidence level for future, which all together keeps the incidence level similar even if the number of days without control increases during the peak.

In contrast, it is observed that the optimal cost and the disease prevalence both are increasing in the non-behavioural model, in either of the delay or gap in the public health initiatives during the outbreak period. This indicates that initiation of public health effort during beginning and late of the outbreak is much more cost-effective than delaying in the beginning, when behavioural interaction is taken as a component of the vaccination dynamics. Hence, our results illustrate that understanding individual vaccinating behaviour is an important component that should be included in public health policy decision-making to control and manage a outbreak.

\subsection{Impact of Declined Risk Perceptions on Vaccination Decision}

We assumed that a new vaccine has been launched for the influenza outbreak. The vaccine has not been used earlier, and nothing much has been known regarding its safety and impact. Under these circumstances, individuals perceived vaccine risk is much higher initially. We have assumed that initially, the perceived risk of the vaccine is $\left(r_{\mathrm{V}}=0.01\right)$ which is much higher than the perceived risk of infection $\left(r_{\mathrm{I}}=0.001\right)$. 


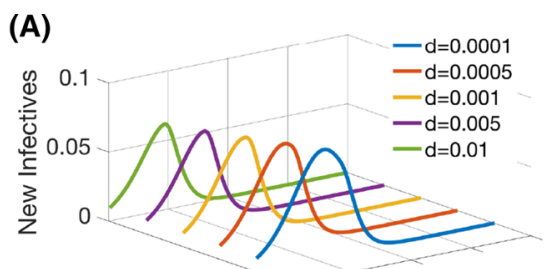

(C)

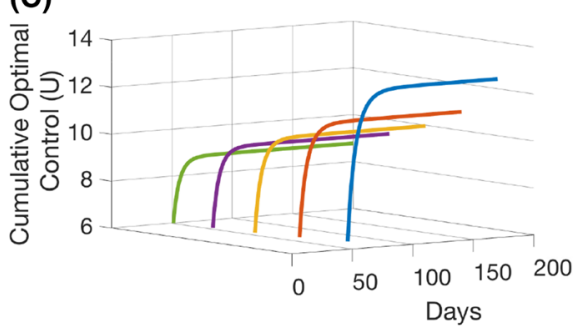

(B)

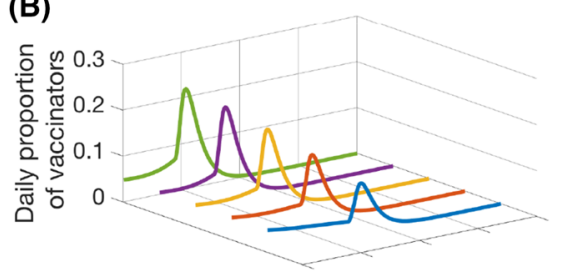

(D)

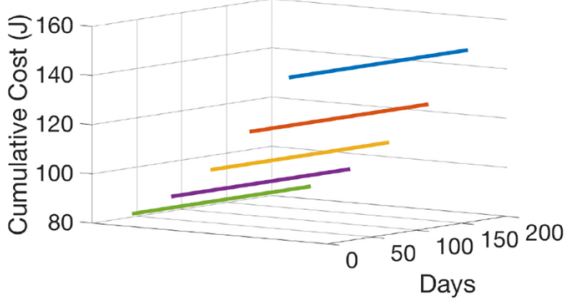

Fig. 9 Dynamics of disease and vaccination under disease death (13). Here, we investigate how with varying disease deaths or hospitalization rates, the disease dynamic changes. The lines in the figure signify varying disease death rate $(d)$ in the population. The control function for behaviour model is taken as square root. a Cumulative infective $\mathbf{b}$ daily proportion of vaccinators, $\mathbf{c}$ cumulative public health effort and $\mathbf{d}$ cumulative cost (absolute value). Here, the parameter regimes are $R_{0}=4, r_{\mathrm{V}}=0.01, r_{\mathrm{I}}=0.001, k=4, \xi=0.95$, $\eta=0.95$. Other parameter values are defined in Table 1

With increasing daily vaccinators, the perception of the vaccine risks declines as it spreads the information in the population about vaccine safety. The parameter $\delta$ defines the sensitivity of reduction due to increase in the vaccination coverage. Figure 8 shows that when the parameter $\delta$ increases from 0 to 15, i.e. the perceived risk of vaccine declines, so vaccination coverage increases $(8 \mathrm{~b})$. As a result, the cumulative number of infective also declines (8a). A similar trend in the dynamics of cumulative effort (8c) and in the total cost of managing the disease (8d) is also observed. This demonstrates a feed-forward mechanism in the model-more people vaccinate, more news of vaccine safety spreads in the population, and this increases the incentives of vaccinations for others and so cumulative vaccine uptake increases. A similar trend in the qualitative dynamics is observed when decline of risk is assumed as linear function of the cumulative vaccine uptake (Fig. S5 in Supplementary information). This, however, signifies the role of public health during the outbreak-they need to act at the beginning of the outbreak, and disseminate information about vaccine safety through more media coverage-rather than putting their effort during the peak.

\subsection{Disease Death from Infection Impacts the Vaccination Behaviour}

Here, we explore how the dynamics of the decision evolves when there is death due to disease severity. Increased death from infection works as an incentive for an individual for vaccination. Figure 9 shows the influence of increased death rates (0.0001-0.01). As shown in Fig. 9b, there is a significant increase in the daily proportion of vaccinators, and lowest new incidence when there is highest death rate $(d=0.01)$ from the 
disease. The interesting observation lies in the optimal dynamics of public health effort (Fig. 9c). It shows that public health program can even delay in the start, while there is death from disease included in the pay-off gain of individual interest (Fig. 9c and d). However, the optimal effort is substantially high in the case of the lowest death rate and so is the total cost from infection and public health effort. So, this result essentially echoes the same what we have described in the last section that dissemination about disease severity at the beginning of the outbreak is way more effective to increase the vaccine uptake, rather than in the middle of the outbreak.

\section{Summary and Conclusions}

Vaccination is one of the most efficient and economic measures to control infections and has been responsible for controlling a large number of diseases (Nichol et al. 1994; Simonsen et al. 2005; Wang et al. 2016; Bauch et al. 2003). But the safety of the vaccine and its associated perceived risks, particularly, in case of a new vaccine, have led to the failure of vaccine uptake (Reluga et al. 2006; Bauch et al. 2010). Under voluntary vaccination, there is always a strategic interaction between individuals whether they decide vaccination or not. Hence, the vaccination coverage depends upon the magnitude of disease prevalence, attainment of herd immunity and the safety of the vaccine (Tchuenche et al. 2011; Bauch et al. 2010; Bhattacharyya and Bauch 2011). Our research highlights that incorporating vaccinating behaviour in disease control models may help policymakers to predict, understand and even reduce the public health cost to manage influenza every year.

Analysis of our game-theoretic model illustrates the optimal allocation of public health effort under different public perceptions. It shows that optimal solutions of vaccination model are significantly different under different public perceptions. Not only this, but also model analysis suggests that policymakers should optimally allocate budget for advertisement and promotion of the vaccine, at the beginning of an outbreak which will validate the safety of the vaccine. Individuals who delayed and refused vaccination were more likely to have concerns on vaccine safety and perceive minimal benefits associated with vaccines. Policymakers may assist providers in responding to individuals who may delay or refuse vaccines. Vaccinating a considerable fraction of the population before a pandemic or seasonal outbreak could significantly bring down the transmission rate. This, however, could be an uphill task for the policymakers due to continuous evolution in virus strain, and particularly relevant to seasonal influenza. More media coverage about vaccine safety, disease severity, even through a social network may increase the visibility of benefits and accelerate the mitigation of epidemics.

Our paper provides an understanding of how public health policymakers can strategize their budget allocation during an influenza outbreak under human vaccination choice. There are certain assumptions we have made in developing our model. We have assumed a deterministic model in the spread of the disease dynamics, but the demographic structure is heterogeneous, and therefore, the behavioural structure may also vary within the population. So, developing an age-specific model may be more realistic in such cases. In this work, we do not identify any vulnerable group in the pop- 
ulation who are at high risk from the infection. In order to make the optimal allocation of limited vaccine supply, policymakers have to target a specific group of population such as children, old people, sick and pregnant women. Targeting populations in the seasonal vaccination would affect the infection rate and also the mortality.

Our model also assumes that the vaccine is perfectly effective, but generally flu vaccines do not have $100 \%$ efficacy (Magpantay et al. 2014). In such case with low efficacy, even vaccinated individuals may get infected, and therefore, the overall burden of disease may not be reduced. Under this circumstance, it remains a challenge to model and find the impact of vaccination behaviour and find the social optimum coverage to mitigate the infection. Lastly, we have assumed the perceived risk of vaccine declines exponentially with increasing uptake of the vaccine. Similarly, we have also considered a linear function for the death rate to characterize its influence on individual perceived risk. However, there are no such data available in the literature in our knowledge. So, a scenario analysis with a different type of functions might worth to understand the influence of behaviour and its impact on vaccine uptake in the population.

Acknowledgements We thank all the reviewers for very useful suggestions and comments that improve the clarity and presentation of the manuscript.

\section{References}

Anderson Roy M, May Robert M (1992) Infectious diseases of humans: dynamics and control. Oxford University Press, Oxford

Arda B, Durusoy R, Yamazhan T, Sipahi OR, Taşbakan M, Pullukçu H, Erdem E, Ulusoy S (2011) Did the pandemic have an impact on influenza vaccination attitude? A survey among health care workers. BMC Infect Dis 11(1):87

Baguelin M, Jit M, Miller E, Edmunds WJ (2012) Health and economic impact of the seasonal influenza vaccination programme in England. Vaccine 30(23):3459-3462

Baguelin M, Flasche S, Camacho A, Demiris N, Miller E, John Edmunds W (2013) Assessing optimal target populations for influenza vaccination programmes: an evidence synthesis and modelling study. PLoS Med 10(10):e1001527

Basta NE, Chao DL, Elizabeth Halloran M, Matrajt L, Longini IM Jr (2009) Strategies for pandemic and seasonal influenza vaccination of schoolchildren in the United States. Am J Epidemiol 170(6):679-686

Bauch CT (2005) Imitation dynamics predict vaccinating behaviour. Proce R Soc B Biol Sci 272(1573):1669-1675

Bauch CT, Bhattacharyya S (2012) Evolutionary game theory and social learning can determine how vaccine scares unfold. PLoS Comput Biol 8(4):e1002452

Bauch CT, Galvani AP, Earn DJD (2003) Group interest versus self-interest in smallpox vaccination policy. Proce Natl Acad Sci 100(18):10564-10567

Bauch CT, Bhattacharyya S, Ball RF (2010) Rapid emergence of free-riding behavior in new pediatric immunization programs. PLoS ONE 5(9):e12594

Bhattacharyya Samit, Bauch Chris T (2011) "wait and see" vaccinating behaviour during a pandemic: a game theoretic analysis. Vaccine 29(33):5519-5525

Bhattacharyya S, Bauch C (2012) Mathematical models of the interplay between individual vaccinating decisions and disease dynamics: a need for closer integration of models and data. Hum Vaccin Immunother $8(6): 842-844$

Bowman Christopher S, Arino J, Moghadas Seyed M (2011) Evaluation of vaccination strategies during pandemic outbreaks. Math Biosci Eng 8(1):113

Brewer NT, Hallman WK (2006) Subjective and objective risk as predictors of influenza vaccination during the vaccine shortage of 2004-2005. Clin Infect Dis 43(11):1379-1386 
Bukhsh A, Rehman H, Hussain Mallhi T, Ata H, Rehman IU, Lee L-H, Goh B-H, Khan TM (2018) Parents' attitude, awareness and behaviour towards influenza vaccination in Pakistan. Hum Vaccin Immunother 14(4):952-957

Carrat F, Flahault A (2007) Influenza vaccine: the challenge of antigenic drift. Vaccine 25(39-40):68526862

Danielle Iuliano A, Roguski Katherine M, Chang Howard H, Muscatello David J, Palekar Rakhee, Tempia Stefano, Cohen Cheryl, Gran Jon Michael, Schanzer Dena, Cowling Benjamin J et al (2018) Estimates of global seasonal influenza-associated respiratory mortality: a modelling study. Lancet 391(10127):1285-1300

Deka A, Bhattacharyya S (2019) Game dynamic model of optimal budget allocation under individual vaccination choice. J Theor Biol 470:108-118

Donaldson LJ, Rutter PD, Ellis BM, Greaves FEC, Mytton OT, Pebody RG, Yardley IE (2009) Mortality from pandemic a/H1N1 2009 influenza in England: public health surveillance study. BMJ 339(b5213):2009

d'Onofrio Alberto, Manfredi Piero, Salinelli Ernesto (2007) Vaccinating behaviour, information, and the dynamics of SIR vaccine preventable diseases. Theor Popul Biol 71(3):301-317

d'Onofrio A, Manfredi P, Poletti P (2011) The impact of vaccine side effects on the natural history of immunization programmes: an imitation-game approach. J Theor Biol 273(1):63-71

d'Onofrio A, Manfredi P, Poletti P (2012) The interplay of public intervention and private choices in determining the outcome of vaccination programmes. PLoS ONE 7(10):e45653

Engler RJM, Nelson MR, Klote MM, VanRaden MJ, Huang C-Y, Cox NJ, Klimov A, Keitel WA, Nichol KL, Carr WW et al (2008) Half-vs full-dose trivalent inactivated influenza vaccine (2004-2005): age, dose, and sex effects on immune responses. Arch Intern Med 168(22):2405-2414

Ferguson NM, Cummings DAT, Fraser C, Cajka JC, Cooley PC, Burke DS (2006) Strategies for mitigating an influenza pandemic. Nature 442(7101):448

Gaff H, Schaefer E (2009) Optimal control applied to vaccination and treatment strategies for various epidemiological models. Math Biosci Eng 6(3):469-492

Galvani AP, Reluga TC, Chapman GB (2007) Long-standing influenza vaccination policy is in accord with individual self-interest but not with the utilitarian optimum. Proc Natl Acad Sci 104(13):5692-5697

GBD 2016 Lower Respiratory Infections Collaborators et al (2018) Estimates of the global, regional, and national morbidity, mortality, and aetiologies of lower respiratory infections in 195 countries, 19902016: a systematic analysis for the global burden of disease study 2016. Lancet Infect Dis 18(11):1191

Gerdil C (2003) The annual production cycle for influenza vaccine. Vaccine 21(16):1776-1779

Hofbauer J, Sigmund K (1998) Evolutionary games and population dynamics. Cambridge University Press, Cambridge

Jones B (2012) Spreading the word about seasonal influenza. Bull World Health Organ 90(4):252-253

Lee S, Golinski M, Chowell G (2012) Modeling optimal age-specific vaccination strategies against pandemic influenza. Bull Math Biol 74(4):958-980

Lenhart S, Workman JT (2007) Optimal control applied to biological models. Chapman and Hall/CRC, Boca Raton

Li M, Chapman GB, Ibuka Y, Lauren AM, Galvani A (2012) Who got vaccinated against H1N1 pandemic influenza? - a longitudinal study in four US cities. Psychol Health 27(1):101-115

Liu W, Ma M-J, Tang F, He C, Zhang X-A, Jiang L-F, Xin D-S, Chun-Yan H, Looman C, Cao W-C (2012) Host immune response to a (H1N1) PDM09 vaccination and infection: a one-year prospective study on six cohorts of subjects. Vaccine 30(32):4785-4789

Louie JK, Acosta M, Winter K, Jean C, Gavali S, Schechter R, Vugia D, Harriman K, Matyas B, Glaser CA et al (2009) Factors associated with death or hospitalization due to pandemic 2009 influenza a (H1N1) infection in California. Jama 302(17):1896-1902

Magpantay FMG, Riolo MA, Domenech De Celles M, King AA, Rohani P (2014) Epidemiological consequences of imperfect vaccines for immunizing infections. SIAM J Appl Math 74(6):1810-1830

Matrajt L, Longini IM Jr (2010) Optimizing vaccine allocation at different points in time during an epidemic. PLoS ONE 5(11):e13767

Medlock J, Meyers LA (2009) Optimizing allocation for a delayed influenza vaccination campaign. PLoS Curr 1

Mercer GN, Barry SI, Kelly H (2011) Modelling the effect of seasonal influenza vaccination on the risk of pandemic influenza infection. BMC Public Health 11(1):S11

Nichol KL, Margolis KL, Wuorenma J, Von Sternberg T (1994) The efficacy and cost effectiveness of vaccination against influenza among elderly persons living in the community. N Eng J Med 331(12):778-784 
Nowak GJ, Sheedy K, Bursey K, Smith TM, Basket M (2015) Promoting influenza vaccination: insights from a qualitative meta-analysis of 14 years of influenza-related communications research by US centers for disease control and prevention (CDC). Vaccine 33(24):2741-2756

Patel R, Longini IM Jr, Elizabeth HM (2005) Finding optimal vaccination strategies for pandemic influenza using genetic algorithms. J Theor Biol 234(2):201-212

Reluga Timothy C (2010) Game theory of social distancing in response to an epidemic. PLoS Comput Biol 6(5):e1000793

Reluga TC, Galvani AP (2011) A general approach for population games with application to vaccination. Math Biosci 230(2):67-78

Reluga TC, Bauch CT, Galvani AP (2006) Evolving public perceptions and stability in vaccine uptake. Math Biosci 204(2):185-198

Rogers CJ, Bahr KO, Benjamin SM (2018) Attitudes and barriers associated with seasonal influenza vaccination uptake among public health students; a cross-sectional study. BMC Public Health 18(1):1131

Schanzer DL, Tam TWS, Langley JM, Winchester BT (2007) Influenza-attributable deaths, Canada 19901999. Epidemiol Infect 135(7):1109-1116

Seale H, Heywood AE, McLaws M-L, Ward KF, Lowbridge CP, Van Debbie C, MacIntyre R (2010) Why do I need it? I am not at risk! public perceptions towards the pandemic (H1N1) 2009 vaccine. BMC Infect Dis 10(1):99

Shahrabani S, Benzion U (2010) Workplace vaccination and other factors impacting influenza vaccination decision among employees in Israel. Int J Environ Res Public Health 7(3):853-869

Shim Eunha (2013) Optimal strategies of social distancing and vaccination against seasonal influenza. Math Biosci Eng 10(5\&6):1615-1634

Simonsen L, Reichert TA, Viboud C, Blackwelder WC, Taylor RJ, Miller MA (2005) Impact of influenza vaccination on seasonal mortality in the US elderly population. Arch Intern Med 165(3):265-272

Strelitz B, Gritton J, Klein EJ, Bradford MC, Follmer K, Zerr DM, Englund JA, Opel DJ (2015) Parental vaccine hesitancy and acceptance of seasonal influenza vaccine in the pediatric emergency department. Vaccine 33(15):1802-1807

Tchuenche JM, Dube N, Bhunu CP, Smith RJ, Bauch CT (2011) The impact of media coverage on the transmission dynamics of human influenza. BMC Public Health 11(1):S5

Tuite AR, Fisman DN, Kwong JC, Greer AL (2010) Optimal pandemic influenza vaccine allocation strategies for the Canadian population. PLoS ONE 5(5):e10520

Ullah R, Zaman G, Islam S (2012) Prevention of influenza pandemic by multiple control strategies. J Appl Math 2012

Verriest E, Delmotte F, Egerstedt M (2005) Control of epidemics by vaccination. In: Proceedings of the 2005, American Control Conference, 2005, pp 985-990. IEEE

Viboud C, Alonso WJ, Simonsen L (2006) Influenza in tropical regions. PLoS Med 3(4):e89

Wang Z, Bauch CT, Bhattacharyya S, d'Onofrio A, Manfredi P, Perc M, Perra N, Salathe M, Zhao D (2016) Statistical physics of vaccination. Phys Rep 664:1-113

Publisher's Note Springer Nature remains neutral with regard to jurisdictional claims in published maps and institutional affiliations.

\section{Affiliations}

\section{Aniruddha Deka $^{1} \cdot$ Buddhi Pantha $^{2} \cdot$ Samit Bhattacharyya ${ }^{1}$}

$\triangle \quad$ Samit Bhattacharyya

samit.b@snu.edu.in

Aniruddha Deka

ad941@snu.edu.in

Buddhi Pantha

bpantha@abac.edu 
1 Disease Modelling Lab, Department of Mathematics, School of Natural Sciences, NH-91, Gautam Buddha Nagar, UP, India

2 College of Arts and Sciences, Abraham Baldwin Agricultural College, Tifton, GA, USA 